\title{
Performance Measurement in Marketing und Verkauf
}

\section{Einsatz von Marketingkennzahlen in den USA und in Kontinentaleuropa}

\author{
Sven Reinecke/ David J. Reibstein
}

Marketing gerät in vielen Unternehmen unter Rechtfertigungsdruck: Der Wert- und Erfolgsbeitrag des Marketing muss ,"messbar" gemacht werden. Eine solche Operationalisierung kann nur über Kennzahlen erfolgen.

- Empirische Ergebnisse belegen, dass Unternehmen in den USA andere Marketingspitzenkennzahlen einsetzen als in Kontinentaleuropa. Ferner integrieren Unternehmen diese Kennzahlen sehr unterschiedlich in die Budgetierungs- und Planungsprozesse sowie in etwaige Management by Objective-Systeme.

- Insgesamt wird deutlich, dass in vielen Unternehmen noch ein großer Nachholbedarf bezüglich der Definition und des Einsatzes von Marketingschlüsselkennzahlen (,„Marketing Metrics" $\left.{ }^{6}\right)$ besteht.

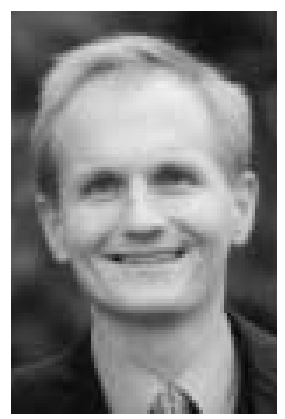

Dr. Sven Reinecke Dozent für Betriebswirtschaftslehre an der Universität St. Gallen (Schweiz) und Leiter des Kompetenzzentrums „Marketingplanung und -controlling" am dortigen Institut für Marketing und Handel.

E-Mail:

sven.reinecke@unisg.ch.

\section{Notwendigkeit eines \\ Marketing Performance Measurement}

Das Schaffen von Werten für Anspruchsgruppen von Unternehmen steht zunehmend im Mittelpunkt der Betriebswirtschaftslehre. Wertorientierte Ansätze fordern eine klare Quantifizierung des Wertbeitrags aller Bereiche und Funktionen. Somit wird auch vom Marketing ein messbarer Wertbeitrag eingefordert, zumal dieser Bereich in vielen Unternehmen zu einem der größten Kostenblöcke geworden ist. Das Marketing hat sich diesen Forderungen bisher häufig dadurch entzogen, dass eine klare Quantifizierung einer marktorientierten Unternehmensführung aufgrund vieler, ,weicher“" Faktoren und unklarer Ursache-Wirkungsbeziehungen nicht möglich oder zumindest wenig sinnvoll sei.

Aufgrund neuerer Forschungsentwicklungen im Bereich des Performance Measurement, die auch die Operationalisierung weicher Variablen umfassen (Stichwort: Scorecard-Ansätze), einer verstärkten Ausrichtung der Controllingpraxis auf

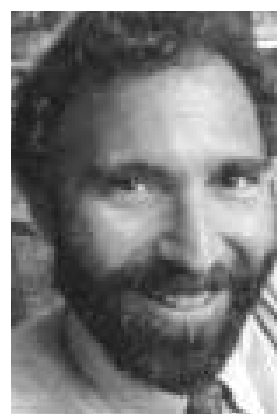

den Unternehmenswert (Stichworte: Shareholder Value, EVA, Discounted Cash flow) sowie modernen Marketingcontrollingansätzen (vgl. Weber/Schäffer 2001) wird die Forderung nach einem messbaren Wertbeitrag des Marketing wieder aktuell.

Ein wichtiges Hilfsmittel, um einen solchen Wertbeitrag herauszuarbeiten, sind Marketingkennzahlen (vgl. hierzu Reinecke 2001). Dabei handelt es sich eigentlich nicht um ein neues, sondern vielmehr um ein sehr traditionelles Thema der Betriebswirtschaftslehre (vgl. Staehle 1967). Auch das Thema, wie man Marketingerfolg messen sollte, beschäftigt Marketingwissenschaftler bereits seit langem (vgl. zum Beispiel Feder 1965). Dennoch waren die bisherigen kennzahlenorientierten Forschungsbemühungen schwerpunktmäßig auf finanzwirtschaftliche Kennzahlen ausgerichtet. Folgende auf Großbritannien bezogene Feststellung von Ambler (2000, S. 1) belegt, dass dies auch in der Praxis der Fall ist: „[...] boards devote nine times more attention to spending and counting cash flow than to wondering where it comes from and how it could be increased" (Ambler 2000, S. 1).

Einige Forschungsprojekte unterstreichen die Aktualität und Relevanz des Themas. So analysierte Davidson (1999), welches die wichtigsten Marketingkennzahlen sein sollten, die im Rahmen von Unternehmensberichten veröffentlicht werden sollten. Clark (1999, S. 712 ff.) arbeitete auf der Basis einer umfangreichen Literaturanalyse drei Entwicklungstrends bezüglich Marketingkennzahlen heraus: Erstens erfolgt eine Entwicklung von finanzwirtschaftlichen zu nichtfinanzwirtschaftlichen Kennzahlen, zweitens von finanzwirtschaftlichen Outputgrößen $\mathrm{zu}$ 
Vorsteuergrößen (insbesondere Kundenzufriedenheit, Kundenloyalität und Brand Equity) und drittens zu einer mehrdimensionalen Messung von Effizienz und Effektivität. Besonders intensiv setzte sich Ambler mit dem Thema Marketingkennzahlen auseinander (vgl. Ambler 2000 und Kokkinaki/Ambler 1999).

Der Bedarf an empirischer Forschung in diesem aktuellen Feld ist allerdings nach wie vor sehr groß. Dies wird beispielsweise auch dadurch deutlich, dass die Partnerunternehmen des renommierten amerikanischen Marketing Science Institute (Cambridge, MA) das Thema „Marketing Metrics“ bereits zum zweiten Mal hintereinander auf Rang 1 der Forschungsprioritäten setzten (vgl. Marketing Science Institute 1998, 2000). Auch Gleich (2001, S. 408) regte einen internationalen Vergleich der Anwendung nichtfinanzieller Kennzahlen und der daraus folgenden Ergebniskonsequenzen an. gebnisse eines internationalen Forschungsprojekts präsentiert. Im Rahmen einer empirischen Studie wurden wesentliche Informationen zum Stand von Marketingplanung und -controlling, eines wertorientierten Marketing Performance Measurements sowie der Arbeit mit Kennzahlen erhoben. Unter anderem wurden folgende Fragen untersucht:
Nachfolgend werden ausgewählte Er-

- Wie gewichten Führungskräfte verschiedene betriebswirtschaftliche Zielbereiche?

- Welche Marketingschlüsselkennzahlen erheben Unternehmen, und welche Bedeutung messen sie diesen bei?

- Wie werden Kennzahlen in den Planungsprozess integriert, und wie zufrieden sind Marketingführungskräfte mit ihren Kennzahlensystemen?

Im Frühsommer 2000 wurde eine standardisierte schriftliche Befragung in der Schweiz, in Deutschland und in den USA durchgeführt (siehe Abbildung 1). Im Rahmen dieser branchenübergreifenden Studie wurden jeweils die auf Geschäftsleitungs- bzw. Vorstandsebene für die Bereiche Marketing und/oder Verkauf Verantwortlichen befragt.

Die Stichprobengrösse war in den drei Ländern unterschiedlich gewählt worden, weil aufgrund von Erfahrungswerten in Deutschland und insbesondere in den USA mit einer im Vergleich zur Schweiz niedrigeren Antwortbereitschaft gerechnet wurde. Die Rücklaufquoten rangieren mit zwischen 10 und 21 Prozent insgesamt in einem für eine branchenübergreifende Studie zu erwartenden und zufriedenstellenden Bereich. Ein Überblick über empirische Studien zum Performance Measurement bei Gleich (2001, S. 104 f. und 264) zeigt, dass die Rücklaufquoten

\begin{tabular}{|c|c|c|c|}
\hline Erhebungszeitraum & \multicolumn{3}{|c|}{ April - August 2000} \\
\hline Art der Studie & \multicolumn{3}{|c|}{ standardisierte schriftliche Befragung } \\
\hline Länder & \multicolumn{3}{|c|}{ deutschsprachige Schweiz, Deutschland, USA } \\
\hline Branchen & \multicolumn{3}{|c|}{$\begin{array}{l}\text { branchenübergreifend (ohne staatliche Unternehmen und Non- } \\
\text { Profit-Organisationen) }\end{array}$} \\
\hline Zielgruppe & \multicolumn{3}{|c|}{$\begin{array}{l}\text { Mitglieder der Geschäfts(bereichs)leitung, die für Marketing } \\
\text { und/oder Verkauf grösserer Unternehmen zuständig sind }\end{array}$} \\
\hline $\begin{array}{l}\text { Befragte } \\
\text { Unternehmen }\end{array}$ & \multicolumn{3}{|c|}{$\begin{array}{l}\text { Schweiz: Zufallsauswahl aus Top } 2000 \text { (Handelszeitung) } \\
\text { Deutschland: Zufallsauswahl aus Top } 2000 \text { (Hoppenstedt) } \\
\text { USA: Zufallsauswahl, Unternehmen bzw. selbständige } \\
\text { Geschäftsbereiche > } 1000 \text { Mitarbeiter (Info USA) } \\
\text { Alle Adressen wurden vorgängig telefonisch überprüft und per- } \\
\text { sonalisiert. }\end{array}$} \\
\hline Methodik & \multicolumn{3}{|c|}{$\begin{array}{l}\text { standardisierte schriftliche Befragung mit einfacher (USA) bzw. } \\
\text { doppelter Mahnwelle ( } \mathrm{CH}, \mathrm{D})\end{array}$} \\
\hline Rücklauf(-quote) & $\begin{array}{l}\text { Schweiz: } \\
\text { Deutschland: } \\
\text { USA: } \\
\text { Total: }\end{array}$ & $\begin{array}{l}n=236 \\
n=182 \\
n=234 \\
n=652\end{array}$ & $\begin{array}{l}21 \text { Prozent } \\
12 \text { Prozent } \\
10 \text { Prozent } \\
15 \text { Prozent }\end{array}$ \\
\hline
\end{tabular}

Abbildung 1: Anlage der empirischen Studie bei solchen schriftlichen Befragungen mit zwischen 3 und 30 Prozent stark variieren. Controllingstudien in den USA weisen zum Teil sogar lediglich Rücklaufquoten von 1,8 oder 2,3 Prozent auf, was in der Regel auf die hohe Anzahl von Befragungen in den Vereinigten Staaten zurückgeführt wird (vgl. Rigby 2001, S. 140 f.). Die absolute Anzahl von über 650 Führungskräften, die sich an der Studie beteiligten, ist allerdings deutlich höher als bei vergleichbaren Untersuchungen.

\section{Priorisierung der Balanced Scorecard- Zielbereiche}

In Anlehnung an das Konzept der Balanced Scorecard von Kaplan/Norton (vgl. 1992, 1996, 2000) wurde das Zielsystem der jeweiligen Geschäftsbereiche mit Hilfe eines Konstantsummenverfahrens abgefragt. Die Befragten wurden gebeten, 100 Punkte auf die vier Kategorien finanzwirtschaftliche Ziele, markt- \& kundengerichtete Ziele, mitarbeiter- \& prozessorientierte Ziele sowie innovationsorientierte Ziele so zu verteilen, dass sie die Zielbedeutung des jeweiligen Geschäftsbereichs bestmöglich charakterisieren. Gemäß dem Konzept der Balanced Scorecard sind diese Ziele nicht unabhängig voneinander; vielmehr bilden sie eine langfristige Ursache-Wirkungsbeziehung ab. Durch die Gewichtung offenbaren die befragten Unternehmen jedoch, welchen Share- bzw. Stakeholdern sie sich besonders verpflichtet fühlen sowie welchen Zeithorizont ihr Zielsystem aufweist; so sind innovationsgerichtete Ziele in der Regel langfristiger als finanzwirtschaftliche Ziele.

Wie aus Abbildung 2 hervorgeht, dominieren in beiden deutschsprachigen Ländern mit 34,4 Prozent die markt- und kundengerichteten Ziele etwas vor den finanzwirtschaftlichen Zielen mit 31,7 Prozent. Mitarbeiter- und prozessorientierte Ziele werden ebenso wie Innovationsziele deutlich niedriger gewichtet. In den USA stehen dagegen die finanzwirtschaftlichen Ziele mit 46,1 Prozent eindeutig im Vordergrund. Markt- und kundenorientierte Ziele folgen mit 29,4 Prozent weit vor den im Vergleich zu Europa noch niedriger gewichteten mitarbeiter- und prozessorien- 


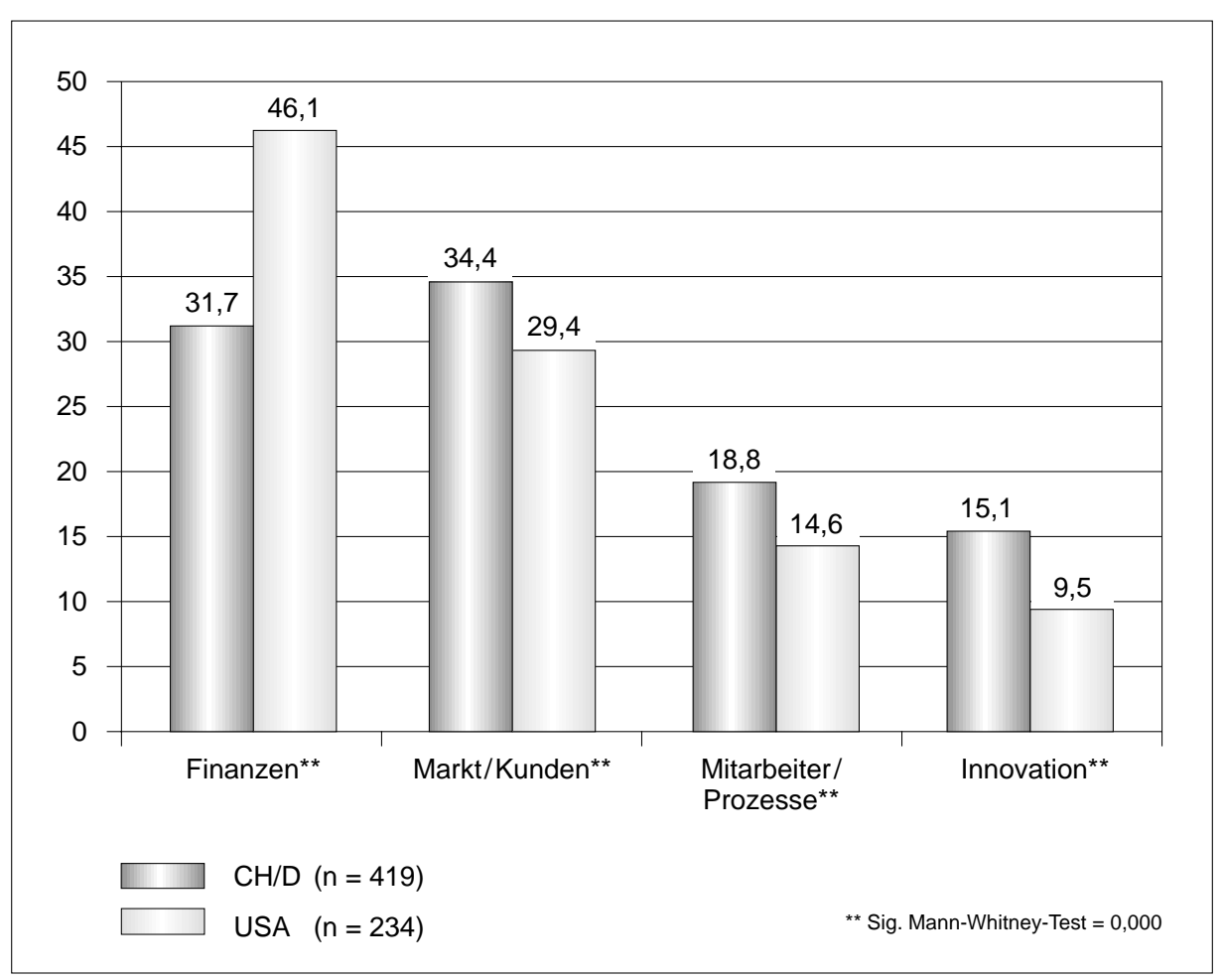

Abbildung 2: Gewichtung der Zielbereiche gemäß dem Konzept der Balanced Scorecard

tierten Zielen (14,6 Prozent) und den Innovationszielen (9,5 Prozent). Überprüft man die Gewichtungen der Zielbereiche mit Hilfe des Mann-Whitney-Tests (aufgrund fehlender Normalverteilung der Daten), so zeigt sich, dass alle Unterschiede höchstsignifikant sind.

Bei der Interpretation dieser Ergebnisse ist allerdings zu berücksichtigen, dass ausschließlich Marketing- und Verkaufsverantwortliche befragt wurden. Es ist $\mathrm{zu}$ vermuten, dass bei einer Befragung von Führungskräften aus den Bereichen Finanzen, Rechnungswesen und Controlling auch in Europa die finanzwirtschaftlichen Ziele stärker als die markt- und kundengerichteten Ziele gewichtet worden wären. Ein analoges Bild ergibt sich, wenn man analysiert, wie Führungskräfte börsennotierter Aktiengesellschaften ihre Ziele gewichten. Unternehmen mit dieser Rechtsform priorisieren selbst in der Schweiz und in Deutschland die finanzwirtschaftlichen Ziele mit 36 Prozent vor den markt- und kundengerichteten Zielen mit 32,1 Prozent. Die Unterschiede zu den USA sind aber ansonsten ähnlich wie in der Gesamtstichprobe, auch wenn sie dies ist etwas, wenn auch nicht bedeutend länger als in den USA (3,2 Jahre). In kaum einem Unternehmen wird über einen Zeitraum von fünf Jahren im voraus geplant.

Die operative Planung richtet sich in den beiden europäischen Ländern im Durchschnitt auf einen Zeitraum von 10,6 Monaten, in den USA auf 9 Monate. Ein operativer Planungshorizont von über einem Jahr ist sowohl in den USA als auch in Europa die absolute Ausnahme.

Lediglich 17,2 Prozent der schweizerischen bzw. deutschen Unternehmen verzichten darauf, zwischen operativer und strategischer Planung zu unterscheiden. In diesen Fällen beträgt der Planungshorizont etwas mehr als ein Jahr. In den USA unterscheiden immerhin gut 30 Prozent der Unternehmen nicht zwischen operativer und strategischer Planung; der durchschnittliche Planungshorizont ist hier mit gut 10 Monaten etwas kürzer als in den beiden europäischen Ländern.

Somit kann festgehalten werden, dass der Planungshorizont im Bereich Marketing und Verkauf in den Vereinigten Staaten zwar statistisch signifikant, aber keineswegs - wie häufig angenommen - beträchtlich kürzer ist als in Europa.

\section{Marketingbudgetierung} den markt- und kundengerichteten Zielen eine niedrigere Signifikanz aufweisen.

Zusammengefasst kann festgestellt werden, dass Führungskräfte aus den Bereichen Marketing und Verkauf in den USA in ihrem jeweiligen Geschäftsbereich eindeutig finanzwirtschaftliche Ziele als vorrangig betrachten, während in Europa die markt- und kundengerichteten Ziele stärker oder zumindest ungefähr gleich stark gewichtet werden.

\section{Planungshorizont in Marketing und Verkauf}

Eine in der Praxis häufig anzutreffende Grundannahme ist, dass der Planungshorizont in den USA deutlich kürzer ist als jener europäischer Unternehmen. Um diese Hypothese $\mathrm{zu}$ überprüfen, wurde die Länge des durchschnittlichen Planungshorizonts erhoben.

In der Schweiz und in Deutschland beträgt die durchschnittliche Dauer des strategischen Planungshorizonts 3,6 Jahre;
Marketingbudgetierung ist ein anspruchsvolles Feld, mit dem sich die Wissenschaft bisher nicht sehr intensiv auseinandergesetzt hat. Welche Methoden werden in der Praxis angewendet, um Marketing- und Verkaufsbudgets festzulegen? Grundsätzlich können sich Budgets an Daten der Vergangenheit orientieren, beispielsweise dem Budget oder den erzielten Umsätzen bzw. Deckungsbeiträgen der Vorperiode. Sie können aber auch aufgrund von qualitativen oder quantitativen Marketingzielen sowie aufgrund von Managementerfahrung festgelegt werden. In der Regel werden Budgets im Rahmen von Aushandlungsverfahren bestimmt, so dass üblicherweise mehrere Verfahren miteinander kombiniert werden. Die befragten Führungskräfte hatten daher die Möglichkeit, aus einer vorgegebenen Liste üblicher Budgetierungsverfahren bis zu drei Methoden anzugeben. Budgetierung in vielen Unternehmen häufig nicht explizit gemacht wird. Mehr
Aus Abbildung 3 geht hervor, dass die 
als die Hälfte aller amerikanischen Unternehmen und immerhin 45 Prozent der Unternehmen aus der Schweiz und Deutschland geben an, dass die Marketing- und Verkaufsbudgets auf Erfahrungswerten basieren. Des Weiteren beeinflussen der angestrebte Umsatz bzw. Absatz - insbesondere in Europa - sowie das Budget der Vorperiode die Budgethöhe. Auch der Umsatz bzw. Absatz der Vorperiode bestimmen in allen drei Ländern häufig das Marketingbudget mit.

Interessanterweise werden sowohl der angestrebte Deckungsbeitrag als auch insbesondere der Deckungsbeitrag der Vorperiode in den USA deutlich stärker zur Budgetierung herangezogen als in den beiden europäischen Ländern (33 im Vergleich zu 23 Prozent bzw. 17 im Vergleich zu 7 Prozent). Dasselbe gilt für den Marktanteil als konkurrenzorientierte Größe, die in den USA immerhin von jedem dritten Unternehmen bei der Budgetierung berücksichtigt wird, in Deutschland und der Schweiz dagegen nur von etwas mehr als einem Fünftel.

Insgesamt kann somit festgestellt werden, dass in der Schweiz und in Deutschland der angestrebte Umsatz, in den USA dagegen der angestrebte Marktanteil als auch angestrebte sowie erzielte Deckungsbeiträge eine größere Rolle spielen. Die Marketingbudgetierung erfolgt somit in den USA etwas differenzierter und insbesondere ergebnisbezogener als in den beiden europäischen Ländern.

\section{Schlüsselkennzahlen in Marketing und Verkauf}

Auf der Basis verschiedener Kategorisierungsschemata von Marketingkennzahlen (vgl. Tomczak et al. 1998 sowie Kokkinaki/Ambler 1999) sowie qualitativer Pretests wurde eine Liste von 23 möglichen Schlüsselkennzahlen für den Bereich Marketing und Verkauf entwickelt. Abbildung 4 zeigt, welche dieser Kenngrößen in der Schweiz und in Deutschland regelmäßig erhoben werden. Zum anderen wurden die Befragten gebeten, bis zu fünf Kennzahlen zu nennen, die für sie besonders relevant sind.

Die Führungskräfte in den beiden europäischen Ländern gaben an, dass Umsatz und Absatz, Nettogewinn, Deckungs-

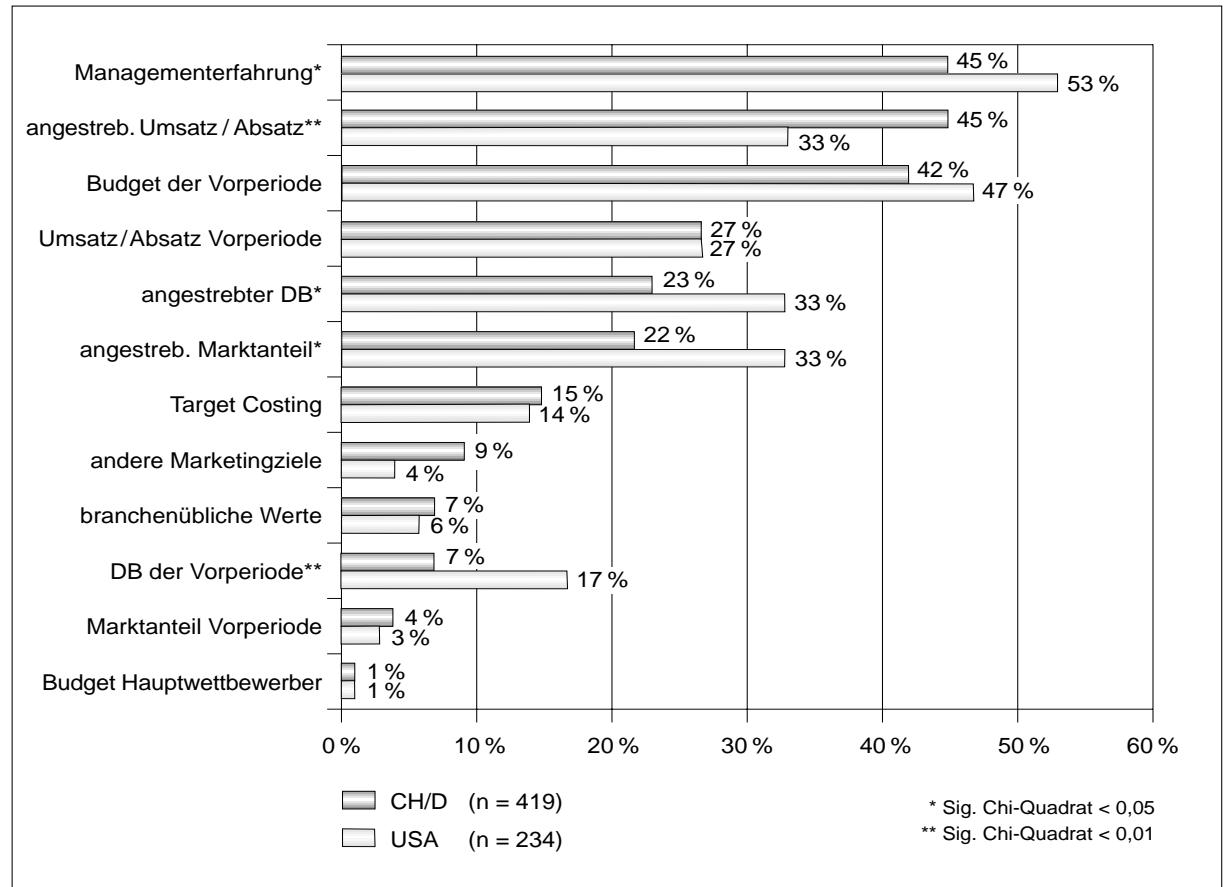

Abbildung 3: Eingesetzte Budgetierungsverfahren in Marketing und Verkauf

beitrag I sowie Umsatzrentabilität jene Kenngrößen sind, die am häufigsten erhoben werden. Aber auch Verhältnisgrößen wie der relative Marktanteil, das relative Umsatzwachstum und der Umsatz pro Mitarbeiter sowie die Kundenzufriedenheit und der erzielte relative Preis werden von mehr als der Hälfte der befragten Unternehmen regelmäßig erhoben. Die Marketingkennzahlen Bekanntheitsgrad und Distributionsgrad werden immerhin noch von etwas mehr als jedem dritten der befragten europäischen Unternehmen regelmäßig gemessen, Kundenbindung und Markenstärke bzw. -wert dagegen nur noch von jedem fünften. Lediglich 17 Prozent der Unternehmen versuchen regelmäßig, den Kundenwert zu bestimmen. Am seltensten werden psychologische Vorgrößen wie Kaufabsicht und Commitment sowie die Kommunikationskennzahl Share of Voice ermittelt.

Berücksichtigt man die Kennzahlenbedeutung, so ergibt sich ein ähnliches Bild: Umsatz bzw. Absatz sind die zentralen Steuerungsgrößen; allerdings ergibt sich eine gewisse Fokusverschiebung auf konkurrenz- und kundenbezogene Größen. So liegen Marktanteil und Kundenzufriedenheit auf den Rängen 3 und 5. Finanzwirtschaftliche Erfolgsgrößen wie
Deckungsbeitrag, Nettogewinn und Umsatzrentabilität werden ebenfalls noch häufig als die zentralen Größen für das Marketing- und Verkaufsmanagement angesehen. Doch lediglich bei jedem zehnten befragten Unternehmen zählt die Kundenbindung zu den fünf wichtigsten Marketingkennzahlen; die Kenngrößen Markenwert bzw. -stärke sowie Kundenwert werden sogar nur von jedem 20. Unternehmen als zentral eingeschätzt.

Abbildung 5 zeigt, welche Marketingkenngrößen in den USA besonders intensiv erhoben und verwendet werden. Auch hier sind Umsatz/Absatz, Nettogewinn und Deckungsbeitrag I die drei am häufigsten erhobenen Kenngrößen. Aber immerhin 73 Prozent der Unternehmen geben an, dass auch das relative Umsatzwachstum sowie der erzielte Preis im Vergleich zur Konkurrenz regelmäßig gemessen werden. Ferner verfolgen deutlich mehr als die Hälfte der befragten Unternehmen regelmäßig den absoluten und relativen Marktanteil, die Umsatzrentabilität sowie die Kundenzufriedenheit. Berücksichtigt man die Bedeutung der Kenngrößen, so bleibt die Rangreihenfolge fast identisch. Lediglich konkurrenzorientierte Größen wie dem relativen Umsatzwachstum und dem im Vergleich zu 


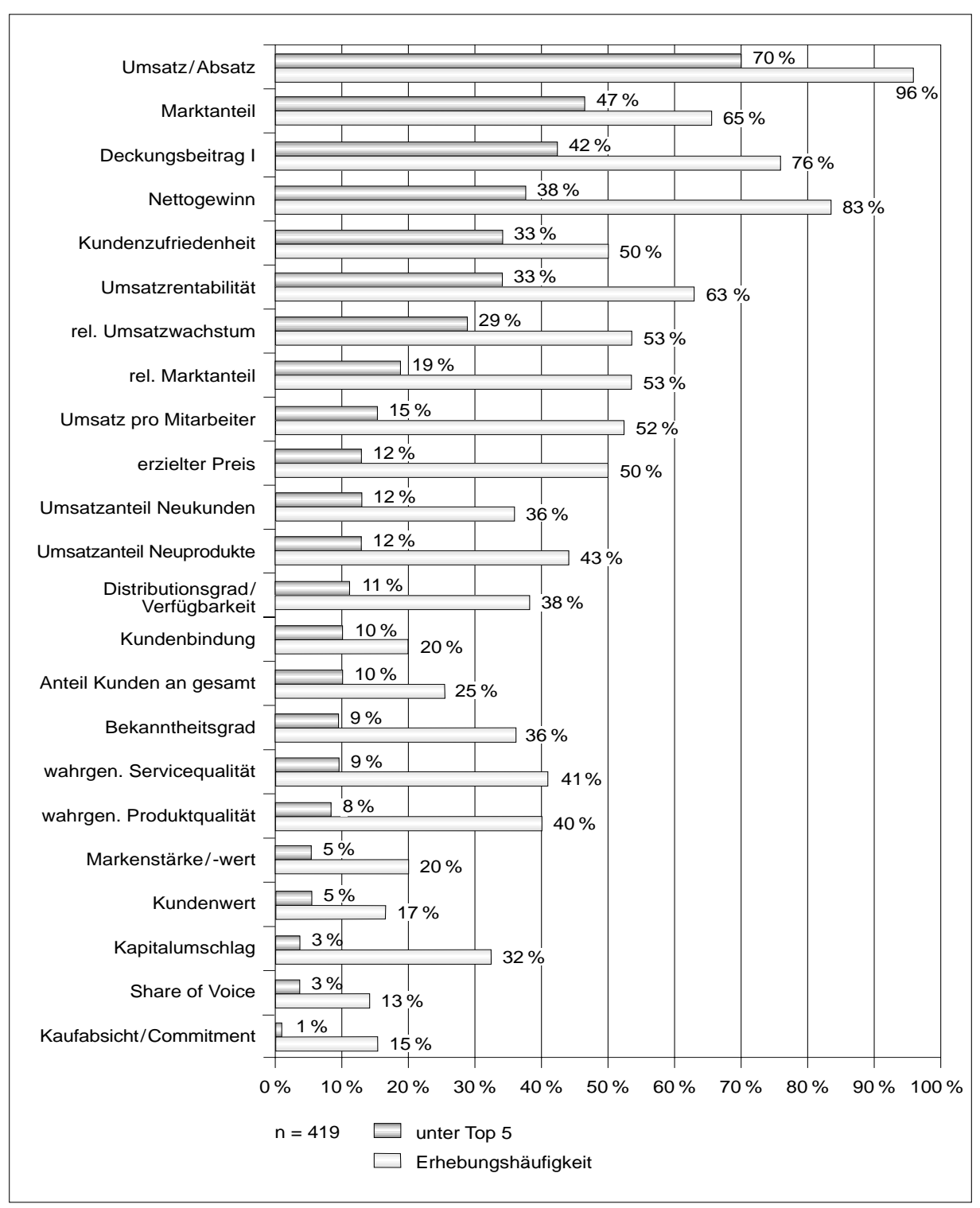

Abbildung 4: Schlüsselkennzahlen in Marketing und Verkauf (CH, D)

den Wettbewerbern erzielten Preis kommen eine etwas geringere Bedeutung zu.

Welche Unterschiede lassen sich zwischen den USA einerseits und der Schweiz und Deutschland andererseits bezüglich der Erhebungsintensität sowie der Bedeutung von Marketingkenngrößen feststellen? In den USA werden insgesamt deutlich mehr Kenngrößen erhoben; die Erhebungsintensität ist bei fast allen Kenngrößen höher. Dies gilt insbesondere für konkurrenzorientierte relative Größen wie das relative Umsatzwachstum und den relativen erzielten Preis sowie für die Kundenbindung (Sig. Chi-Quadrat $<0,01$ ).
Mit Hilfe loglinearer Modelle konnte allerdings gezeigt werden, dass die signifikanten Unterschiede zwischen den USA und Europa bei einigen Variablen primär auf Branchen- und/oder Rechtsformunterschiede der zwei Stichproben zurückzuführen sind - und weniger auf Kontinentunterschiede. (So ist der Anteil von Aktiengesellschaften und Dienstleistungsunternehmen in den USA etwas höher.) Bestätigt werden konnte allerdings, dass die Größen Nettogewinn, Deckungsbeitrag I, relatives Umsatzwachstum, erzielter relativer Preis, Anteil Kunden an potentiellen Gesamtkunden und Kundenbindung in den USA deutlich häufiger erhoben werden, während die Kennzahl Umsatz pro Mitarbeiter in Deutschland und der Schweiz häufiger berechnet wird.

Ein etwas anderes Bild zeigt sich, wenn man die Bedeutung der Kenngrößen in den USA sowie in Europa vergleicht. Deckungsbeitrag und Nettogewinn rangieren in den USA in der Bedeutungsskala etwas höher als in Europa, während die europäischen Führungskräfte der klassischen Kenngröße „Umsatzrentabilität" einen deutlich höheren Stellenwert als ihre amerikanischen Kollegen einräumen. Auch wenn Markenstärke und -wert sowie Kundenwert in allen Ländern nur selten zu den wichtigsten Kenngrößen zählen, so fällt doch auf, dass knapp dreimal so viele Unternehmen in den USA den Markenwert bzw. die Markenstärke unter die fünf wichtigsten Marketingkennzahlen einordnen. Diese Abhängigkeiten zwischen Kontinent und Kennzahlenbedeutung erwiesen sich auch bei Berücksichtigung von Branchen- und Rechtsformunterschieden als signifikant.

Zusammenfassend kann festgestellt werden, das Unternehmen in den Vereinigten Staaten wesentlich mehr Kennzahlen erheben als europäische Unternehmen. Ferner fokussieren sie deutlich stärker auf die ergebnisbezogenen Kenngrößen Nettogewinn und Deckungsbeitrag I, während europäische Unternehmen die „klassischen“ Verhältniskennzahlen wie Umsatzrentabilität und Umsatz pro Mitarbeiter bevorzugen. Insgesamt stehen aber sowohl in den USA als auch Europa selbst für Marketing- und Verkaufsleiter finanzwirtschaftliche Kennzahlen im Vordergrund. Lediglich den Größen Marktanteil und Kundenzufriedenheit kommt noch eine gewisse Bedeutung $\mathrm{zu}$, während differenzierteren Marketingkennzahlen wie Kunden- und Markenwert kaum Beachtung geschenkt wird.

\section{Zufriedenheit mit dem Marketingberichtssystem}

Weder die Führungskräfte in den USA noch in Deutschland oder der Schweiz sind mit den Berichtssystemen in den Bereichen Marketing und Verkauf besonders zufrieden (siehe Abbildung 6). Sowohl der konzeptionelle Aufbau der Systeme 
als auch die informationstechnische Unterstützung und erst recht die Integration externer Daten und Informationen in das Berichtssystem werden als nicht zufriedenstellend betrachtet. Auch entsprechen sie häufig nicht den Zeitanforderungen des Managements.

Bei einer durchgeführten Regressionsanalyse offenbarte das hohe angepasste Bestimmtheitsmaß $\left(\mathrm{R}^{2}\right)$ von 0,702 für Europa und von sogar 0,890 für die USA, dass die erhobenen Teilzufriedenheiten die Gesamtzufriedenheit mit den Berichtssystemen sehr gut erklären. In den beiden europäischen Ländern hat dabei der konzeptionelle Aufbau des Berichtssystems den weitaus grössten Einfluss auf die Gesamtzufriedenheit; in den USA kommt der informationstechnologischen Unterstützung eine ebenso starke Rolle zu.

Insgesamt ist die Unzufriedenheit mit dem Marketing- und Verkaufsberichtswesen in den USA im Durchschnitt noch etwas größer als in den beiden europäischen Ländern. Daraus kann allerdings nicht gefolgert werden, dass die Berichtssysteme in den USA eine niedrigere Qualität aufweisen; vielmehr könnte auch das Anspruchsniveau der Führungskräfte höher sein. Dieses Ergebnis unterstreicht den Handlungsbedarf im Bereich des Marketingcontrollings bzw. Marketing Performance Measurements.

\section{Stellenspezifische Ziele}

Der Führungskreislauf schließt sich nur dann, wenn sich die aus Sicht des Managements relevanten betriebswirtschaftlichen Spitzenkennzahlen bzw. Marketingschlüsselkenngrößen in stellenspezifischen Zielvereinbarungen niederschlagen, beispielsweise im Rahmen eines Management by Objectives. Um dies zu überprüfen, wurden die Führungskräfte mit Hilfe einer offenen Frage gebeten, die wichtigsten Ziele ihrer eigenen Stelle zu nennen (maximal vier) und deren Bedeutung prozentual zu gewichten. Diese qualitativen Antworten wurden kodiert, das heißt, jedes Ziel wurde einer von insgesamt 23 möglichen Zielkategorien zugeordnet. Abbildung 7 zeigt den Anteil jeder Zielkategorie an der Gesamtzahl der genannten Ziele sowie die jeweilige durchschnittliche Zielgewichtung.

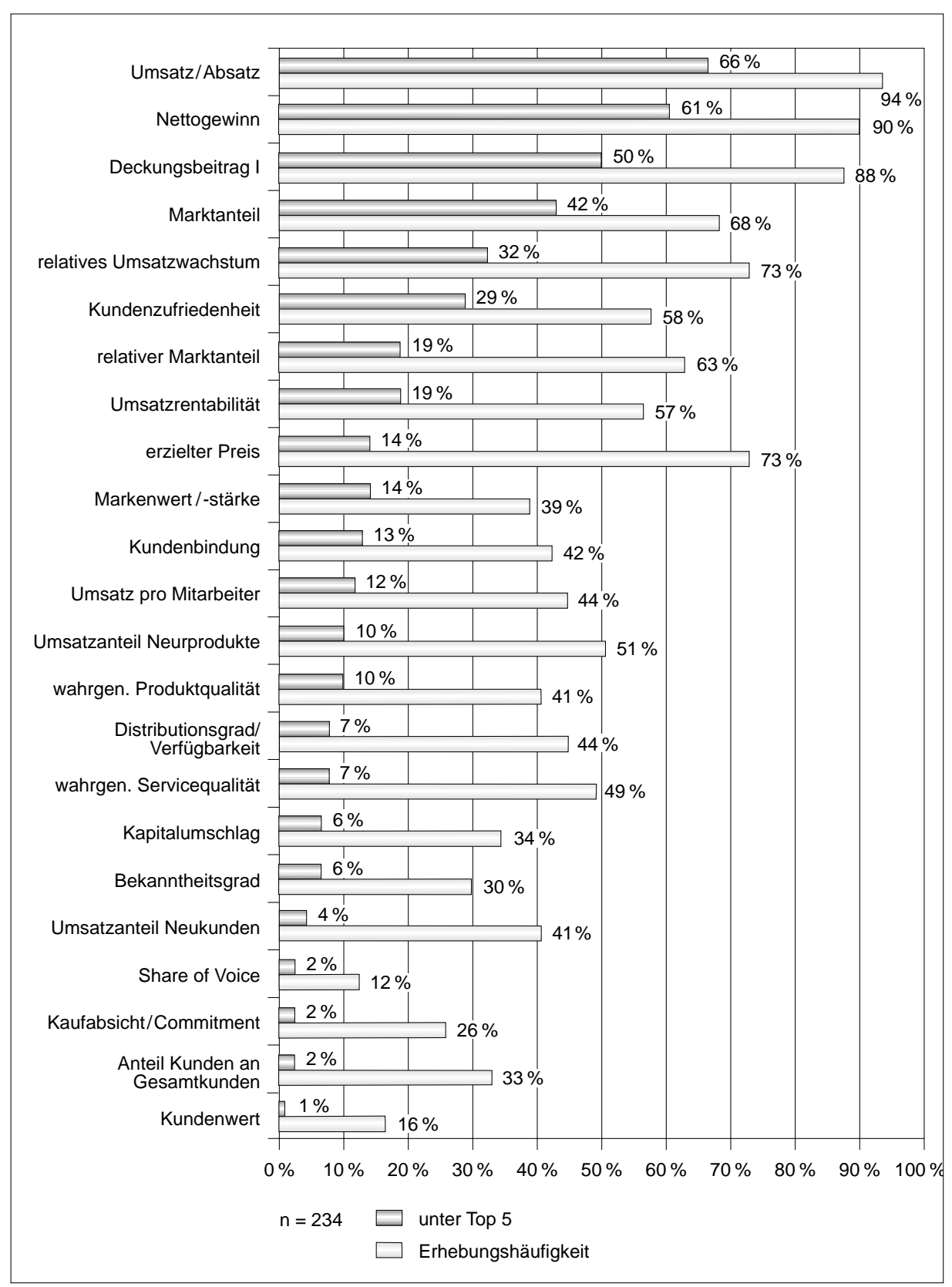

Abbildung 5: Schlüsselkennzahlen in Marketing und Verkauf (USA)

Insgesamt zeigt sich, dass finanzwirtschaftliche Ziele sowohl in den USA als auch in Europa am häufigsten genannt und auch am stärksten gewichtet werden. Dabei handelt es sich neben den klassischen Mengenzielen (Umsatz, Absatz) fast ebenso häufig um Ergebnisziele. Interessant ist, dass der Anteil finanzwirtschaftlicher Kenngrößen bei den Stellenzielen in der Schweiz und in Deutschland noch höher ist als in den USA. Dies scheint der in Ab- schnitt 2 dargestellten eindeutigen Priorisierung der finanzwirtschaftlichen Ziele in den USA zu widersprechen. Doch kann es andererseits auch ein Indiz dafür sein, dass Management by Objective-Systeme in den USA häufig bereits etwas differenzierter sind als in Europa, indem sie versuchen, mehrere Zielbereiche abzudecken. So werden in den USA beispielsweise konkrete kunden- und marktspezifische Kennzahlen häufiger eingesetzt. 


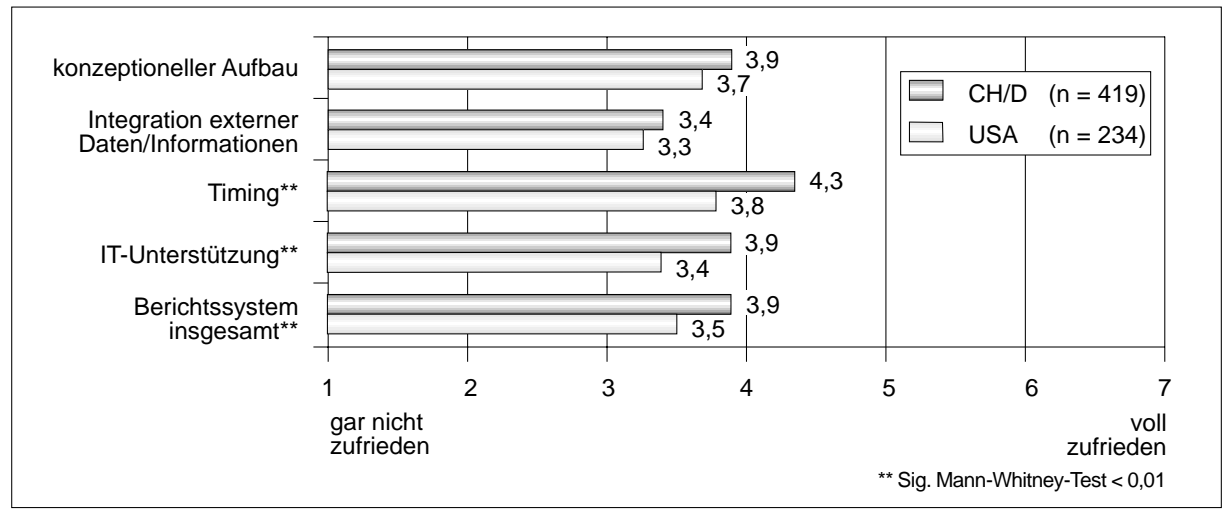

Abbildung 6: Zufriedenheit mit dem Berichtssystem in Marketing \& Verkauf

\begin{tabular}{|c|c|c|c|c|}
\hline $\begin{array}{l}\text { Stellenspezifische Ziele } \\
\text { CH/D: } n=1111 \\
\text { USA: } n=680\end{array}$ & $\begin{array}{l}\mathrm{CH} / \mathrm{D} \\
\text { Nennung } \\
\%\end{array}$ & $\begin{array}{l}\text { USA } \\
\text { Nennung } \\
\%\end{array}$ & $\begin{array}{l}\mathrm{CH} / \mathrm{D} \\
\text { Gewich- } \\
\text { tung \% }\end{array}$ & $\begin{array}{l}\text { USA } \\
\text { Gewich } \\
\text { tung \% }\end{array}$ \\
\hline finanzwirtschaftliche Ziele (total) & 45,6 & 41,6 & 33 & 34 \\
\hline finanzwirtschaftliche Ziele (allgemein) & 3,6 & 2,9 & 26 & 27 \\
\hline Umsatz-/Absatzziele & 18,7 & 15,7 & 35 & 35 \\
\hline Ergebnisziele & 17,0 & 17,9 & 34 & 35 \\
\hline Kostenziele/budgetbezogene Ziele & 3,7 & 2,8 & 24 & 24 \\
\hline kapitalmarktbezogene Ziele & 0,2 & 0,9 & 20 & 31 \\
\hline sonstige finanzwirtschaftliche Ziele & 2,4 & 1,3 & 42 & 37 \\
\hline kunden-/marktbezogene Ziele (total) & 30,9 & 35,7 & 27 & 27 \\
\hline kunden-/marktbezogene Ziele (allgemein) & 3,3 & 3,4 & 30 & 31 \\
\hline konkurrenzbezogene Ziele/Marktanteilsziele & 6,0 & 6,2 & 26 & 28 \\
\hline übergreifende kundenbezogene Ziele & 0,5 & 0,0 & 22 & 0 \\
\hline Einstellungsziele, Markenwert/-stärke & 3,9 & 5,7 & 26 & 26 \\
\hline Kundenakquisitionsziele & 5,0 & 4,4 & 29 & 29 \\
\hline Kundenbindungsziele & 7,4 & 6,9 & 28 & 28 \\
\hline marktpartnerbezogene Ziele & 0,3 & 0,9 & 33 & 33 \\
\hline produktbezogene Marketingziele & 3,7 & 6,8 & 26 & 23 \\
\hline kommunikationsbezogene Marketingziele & 0,7 & 1,3 & 17 & 21 \\
\hline mitarbeiter-/prozessbezogene Ziele (total) & 14,5 & 11,8 & 22 & 23 \\
\hline mitarbeiter-/prozessbezog. Ziele (allgemein) & 1,2 & 1,9 & 23 & 20 \\
\hline mitarbeiterbezogene Ziele & 2,7 & 1,2 & 21 & 21 \\
\hline Kompetenzverbesserungsziele & 1,3 & 2,8 & 20 & 23 \\
\hline informationsbezogene Ziele & 5,8 & 3,4 & 22 & 24 \\
\hline nichtspezifizierte Abteilungs-/Bereichsziele & 1,6 & 1,6 & 27 & 20 \\
\hline nichtspezifizierte Individualziele & 1,9 & 0,9 & 33 & 29 \\
\hline Innovationsziele (total) & 6,6 & 8,5 & 22 & 22 \\
\hline Innovationsziele (allgemein) & 5,6 & 4,3 & 22 & 22 \\
\hline Entwicklungsziele & 0,4 & 2,8 & 20 & 20 \\
\hline Markteinführungsziele & 0,6 & 1,5 & 26 & 22 \\
\hline nicht zuordenbare Ziele & 2,4 & 2,4 & 28 & 39 \\
\hline
\end{tabular}

Abbildung 7: Zielkategorien der genannten stellenbezogenen Ziele
Eine Clusteranalyse (siehe Abbildung 8) der stellenspezifischen Ziele von Marketingführungskräften deutscher und schweizerischer Unternehmen belegt, dass zahlreiche Unternehmen ausschließlich finanzwirtschaftliche Ziele in ihre Führungssystem integrieren (Cluster 2). Ungefähr ebenso viele setzen ausgewogene Ziele und betonten insbesondere innovationsorientierte Ziele deutlich (Cluster 1). Die größte Gruppe kombiniert primär finanzwirtschaftliche und kunden- bzw. marktorientierte Ziele (Cluster 3), vernachlässigt allerdings die Innovationsziele.

Überhaupt verwenden sehr wenige Unternehmen Innovationsziele; insbesondere gibt es kaum Ziele, die sich konkret auf den Markteinführungsprozess neuer Leistungen beziehen. Zukunftsbezogene Potentialkennzahlen werden somit - zumindest für Marketing- und Verkaufsführungskräfte - kaum als Zielvorgaben verwendet.

Des Weiteren ist festzustellen, dass kompliziertere Marketingkennzahlen wie beispielsweise Einstellungsgrößen oder Markenstärke bzw. -wert kaum als konkrete Stellenvorgaben zum Einsatz kommen. Daher ist zu vermuten, dass die meisten Unternehmen aus Praktikabilitätsgründen auf sehr einfach zu messende, harte und eindeutige Ziele zurückgreifen. Werden Führungskräfte danach beurteilt, wie stark sie diese Ziele erreichen, und wird gar ihre Vergütung daran gekoppelt, so resultiert daraus, dass sie auch diese „einfachen“ Ziele priorisieren werden. Dies macht deutlich, dass die Marketingforschung zwar zahlreiche Kennzahlen entwickelt und ihre Operationalisierung optimiert hat, diese im Planungs- und Controlling-Prozess in der Realität aber dennoch nur sehr selten eingesetzt werden. Somit besteht in diesem Bereich eine eindeutige Implementierungslücke. Ein Grund hierfür könnte in dem Trugschluß liegen, dass harte Ziele „gerechter" sind, weil sie ursächlicher von den jeweiligen Führungskräften beeinflußt werden können. Häufig ist das Gegenteil richtig - so wäre es sinnvoller, einen Marketingleiter beispielsweise nach Einstellungszielen wie Kundenzufriedenheit oder Markenstärke zu beurteilen, weil er diese direkter beeinflussen kann als die häufig bevorzugten Umsatz- und Ergebnisziele (vgl. Reinecke 2001).

In einigen Unternehmen wird das Entgelt von Führungskräften an die Zielerrei- 
chung gekoppelt. $\mathrm{Zu}$ vermuten ist, dass in den USA deutlich stärker erfolgsabhängig vergütet wird als in Europa. Die empirischen Ergebnisse bestätigen dies eindrücklich und hochsignifikant (Sig. Mann-Whitney-Test $=0,000$ ): Im Durchschnitt sind die Gehälter in Deutschland und der Schweiz zu 85 Prozent fix; somit hängen lediglich 15 Prozent des Entgelts vom Erfolg ab. In den USA beträgt das Verhältnis dagegen 74 zu 26 Prozent, das heißt, dass bei Marketing- und Verkaufsführungskräften in den USA mehr als ein Viertel des Entgelts erfolgsabhängig ist.

Zusammenfassend kann festgestellt werden, dass im Rahmen von stellenspezifischen Zielen sowohl in den USA als auch in der Schweiz und in Deutschland hauptsächlich einfache Kenngrößen eingesetzt werden. Insgesamt unterscheiden sich die Länder nicht maßgeblich voneinander, wenngleich die Zielsysteme in den USA entgegen den ursprünglichen Annahmen etwas ausgeglichener als in Europa sind, das heißt, häufiger nicht nur finanzwirtschaftliche, sondern auch konkrete kundenund marktbezogene Ziele berücksichtigen. Die Erfolgsabhängigkeit des Entgelts von Marketing- und Verkaufsführungskräften ist dagegen in den USA deutlich und hochsignifikant höher als in Europa.

\section{Ausblick}

Die explorativen Ergebnisse der vorliegenden Studie bieten erstmals einen systematischen Vergleich zwischen dem Einsatz von Marketingkennzahlen in den USA einerseits und zwei kontinentaleuropäischen Ländern andererseits. Aufgrund der Untersuchungen von Kokkinaki/Ambler (1999) und Ambler/Riley (2000) sind allerdings Unterschiede zwischen den deutschsprachigen Ländern und Großbritannien zu vermuten, so dass weitere internationale Forschungsprojekte möglichst immer sowohl kontinentaleuropäische Länder als auch Großbritannien einschließen sollten.

Die vorliegende Untersuchung hat offenbart, dass deutliche Diskrepanzen zwischen den in der Wissenschaft entwickelten Marketingkenngrößen und ihrem Einsatz in der Praxis festzustellen sind. So werden die Grössen Markenwert bzw. -stärke sowie Kundenwert in Europa nur von jedem 20. Unternehmen als zentral

\begin{tabular}{|l|c|c|c|}
\hline Final Cluster Centers (CH/D) & $\begin{array}{c}\text { Cluster 1 } \\
(\mathrm{n}=76)\end{array}$ & $\begin{array}{c}\text { Cluster 2 } \\
(\mathrm{n}=71)\end{array}$ & $\begin{array}{c}\text { Cluster 3 } \\
(\mathrm{n}=180)\end{array}$ \\
\cline { 2 - 4 } & $\begin{array}{c}\text { „Die Aus- } \\
\text { gewogenen“ }\end{array}$ & $\begin{array}{c}\text { „Die Fianz- } \\
\text { orientierten“" }\end{array}$ & $\begin{array}{c}\text { "Die Tradi- } \\
\text { tionellen“ }\end{array}$ \\
\hline finanzwirtschaftliche Ziele & 0.42 & 0.99 & 0.87 \\
\hline kunden-/marktorientierte Ziele & 0.99 & 0.00 & 0.81 \\
\hline mitarbeiter-/Prozessbezogene Ziele & 0.33 & 0.00 & 0.33 \\
\hline innovationsorientierte Ziele & 0.80 & 0.10 & 0.02 \\
\hline sonstige Ziele & 0.08 & 0.13 & 0.04 \\
\hline
\end{tabular}

Abbildung 8: Clusteranalyse stellenspezifischer Zielsetzungen (CH/D)

eingeschätzt - und dies, obwohl die Marketingwissenschaft fast unisono gerade Markenwert (vgl. Keller 2000; Ambler 2000) oder Kundenwert (Rust/Zeithaml/ Lemon 2000; Günter/Helm 2001) als absolute Topkennzahlen bezeichnet. Marketingwissenschaft und Praxis des Marketingcontrollings klaffen hier weit auseinander: Führungskräfte in der Praxis sollten überprüfen, ob sie nicht vermehrt differenziertere Marketing- und Verkaufskenngrößen nutzbringend einsetzen könnten. Gleichzeitig ist die Wissenschaft herausgefordert zu analysieren, wieso die Erkenntnisse in diesem Gebiet auf so große Implementierungsprobleme stoßen.

\section{Literaturhinweise}

Ambler, T.: Marketing and the Bottom Line - The New Metrics of Corporate Wealth, London 2000.

Amber, T./Kokkinaki, F.: Measures of Marketing Success, in: Journal of Marketing Management (1997), 13, S. 665-678.

Amber, T./Kokkinaki, F.: Marketing Performance Assessment: An Exploratory Investigation into Current Practice and the Role of Firm Orientation, Working Paper Report, H. 99-114, Marketing Science Institute, Cambridge (Massachusetts) 1999.

Ambler, T./Riley, D.: Marketing Metrics: A Review of Performance Measures in Use in the UK and Spain, Working Paper Report, H. 00-500, Marketing Science Institute, Cambridge (Massachusetts) 2000.

Clark, B. H.: Marketing Performance Measures: History and Interrelationships, in: Journal of Marketing Management (1999), 15, S. 711-732.

Davidson, J. H.: Transforming the Value of Company Reports through Marketing Measurement, in: Journal of Marketing Management (1999), 15, S. 757-777.

Ehrbahr, A.: EVA - The Real Key to Creating Wealth, New York et al. 1998.

Feder, R. A.: How to Measure Marketing Performance, in: Harvard Business Review (1965(, 43, H. 3, S. 132-142.
Gleich, R.: Das System des Performance Measurement: Theoretisches Grundkonzept, Entwicklungs- und Anwendungsstand, München 2001.

Günter, B./Helm, S. (Hrsg.): Kundenwert: Grundlagen - Innovative Konzepte - Praktische Umsetzungen, Wiesbaden 2001.

Kaplan, R.S./Norton, D.P.: The Balanced Scorecard - Measures that Drive Performance, in: Harvard Business Review (1992), 70, H. 1, S. 71-79.

Kaplan, R.S./Norton, D.P.: The Balanced Scorecard, Translating Strategy into Action, Boston (Massachusetts) 1996.

Kaplan, R.S./Norton, D. P.: Having Trouble with Your Strategy - Then Map it, in: Harvard Business Review (2000), 78, H. 4, S. 3-11.

Keller, K.L.: The Brand Report Card, in: Harvard Business Review (2000), 78, H. 1, S. 147-157.

Marketing Science Institute: Research Priorities 1998 - 2000, Cambridge (Massachusetts) 1998.

Marketing Science Institute: Research Priorities 2000 - 2002, Cambridge (Massachusetts) 2000.

Rigby, D.: Management Tools and Techniques: A Survey, in: California Management Review (2001), 43, H. 2, S. 139-160.

Reinecke, S.: Marketing-Kennzahlensysteme: Notwendigkeit, Gütekriterien und Konstruktionsprinzipien, in: Reinecke, S./Tomczak, T./Geis, G. (Hrsg.): Handbuch Marketingcontrolling, St. Gallen/Wien: Thexis/Ueberreuter, 2001, S. 690719.

Rust, R.T./Zeithaml, V.A./Lemon, K. N.: Driving Customer Equity - How Customer Lifetime Value is Reshaping Corporate Strategy, New York et al. 2000.

Staehle, W. H.: Kennzahlen und Kennzahlensysteme, Ein Beitrag zur modernen Organisationstheorie, Diss., München 1967.

Tomczak, T./Reinecke, S./Karg, M./Mühlmeyer, J.: Best Practice in Marketing - Empirische Erfolgsstudie zum aufgabenorientierten Ansatz, Fachbericht für Marketing, H. 2, St. Gallen 1998.

Treasurer's Department of E. I. DuPont de Nemours and Company: Executive Committee Control Charts, 4. Auflage (1. Auflage: 1919), Wilmington (Delaware) 1959.

Weber, J./Schäffer, U.: Marketingcontrolling: Sicherstellung der Rationalität in einer marktorientierten Unternehmensführung, in: Reinecke, S./Tomczak, T./Geis, G. (Hrsg.): Handbuch Marketingcontrolling, St. Gallen/Wien: Thexis/Ueberreuter, 2001, S. 32-49.

\section{Sven Reinecke/}

David J. Reibstein 


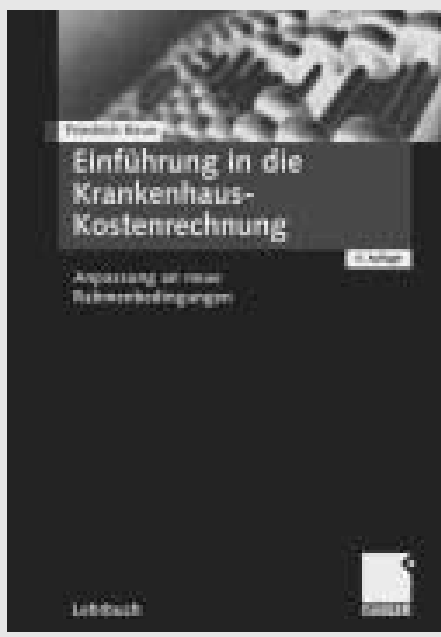

Friedrich Keun führt systematisch in die Krankenhaus-Kostenrechnung, insbesondere in die Kostenarten-, Kostenstellen- und Kostenträgerrechnung, ein. Er verknüpft allgemeine Grundlagen mit krankenhausspezifischen Problemen. Eingehend werden die konkreten Auswirkungen des augenblicklichen Entgeltsystems analysiert. Die vierte Auflage berücksichtigt darüber hinaus die zukünttigen Anforderungen an die Krankenhaus-Kostenrechnung, indem ausführlich die Diagnosis Related Groups (DRGs) beschrieben werden, deren Einführung als KrankenhausEntgeltsystem im J ahr 2003/2004 bevorsteht.

Friedrich Keun $\mathrm{Br}, € 31,00$
Einführung in die Krankenhaus-Kostenrechnung Anpassung an neue Rahmenbedingungen 4., überarb. Aufl. 2001. XX, $226 \mathrm{~S}$. ISBN 3-409-42908-5

\author{
Beate Kremin-Buch \\ Strategisches \\ Kostenmanagement \\ Grundlagen und \\ moderne Instrumente. \\ Mit Fallstudien. \\ 2., vollst.überarb. Aufl. \\ 2001. XIV, $226 \mathrm{~S}$ \\ $B r . € 26,00$ \\ ISBN 3-409-22266-9
}

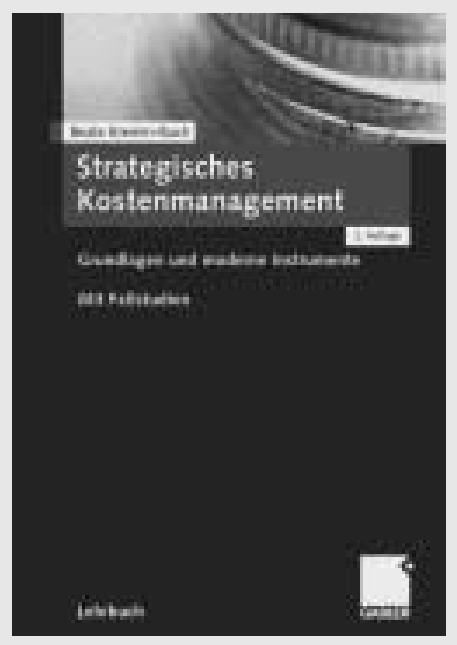

Beate Kremin-Buch stellt mit dem Fixkostenmanagement, der Prozesskostenrechnung, dem Target Costing, dem Product Lifecycle Costing sowie dem Cost Benchmarking die Kerninstrumente des strategischen Kostenmanagement klar und verständlich dar. Besonderen Wert legt die Autorin darauf, die Instrumente nicht nur isoliert zu beschreiben, sondern auch deren Beziehung zueinander zu verdeutlichen. Zahlreiche Fallstudien erleichtern das Verständnis. Für die zw eite Auflage wurde das "Strategische Kostenmanagement" vollständig überarbeitet. Neue Praxisbeispiele veranschaulichen und vertiefen die inhaltlichen Zusammenhänge.

\section{Bestellung} Fax: $0 \overline{6} \overline{11} . \overline{78} \overline{78}-4 \overline{20}$

Ja, ich bestelle:

Friedrich Keun Expl. Einführung in die KrankenhausKostenrechnung $B r . € 31,00$ ISBN 3-409-42908-5

Vorname und Name

\section{Straße (bitte kein Postfach)} Expl. Strategisches Kostenmanagement Br.€ 26,00 ISBN 3-409-22266-9
PLZ, Ort

Unterschrift

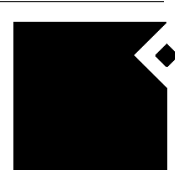

GABLER 